\title{
DESIGN CENTRIFUGAL PUMP ASSISTED BY CF TURBO 10.2 SOFTWARE
}

\author{
Siprianus Ndaha Ate \\ Department of Mechanical Engineering, Faculty of Engineering, University of Merdeka Malang \\ Email corresponding author: prytosandaate@gmail.com
}

\begin{abstract}
In the industrial world the use of centrifugal pumps is often used to distribute water that is to support the smooth running of industrial processes or to cool a machine. Centrifugal pumps have a very broad scope of use with the head and the resulting capacity is very large and varied. In this final project, a centrifugal pump with a capacity of $30 \mathrm{~m} 3$ / hour, a pump head of 10 meters, a shaft rotation of $1500 \mathrm{rpm}$ and a pressure difference of $10 \mathrm{bar}$ in and out is planned. In the planning of centrifugal pumps, CF Turbo 10.2 software is used. Fluid is water ("Water") with a temperature of $200 \mathrm{C}$. Specific gravity of $1 \mathrm{~kg} / \mathrm{dm} 3$. The output of the software includes blade diameter, shape, number of blades and geometric representation of blades. In planning this centrifugal pump as a comparison material, the specifications of the centrifugal pump are available. 5-level centrifugal pump is planned, each level has a head of 10 meters, a discharge of $30 \mathrm{~m} 3$ / hour.
\end{abstract}

Keywords : Centrifugal Pump, Impeller, CF Turbo Software 10.2

\section{INTRODUCTION}

\section{Background}

The water pump is a means of transporting liquid fluids, which is a tool commonly used in everyday life. Its benefits in helping to complete the transfer of water/liquid fluid make water pumps commonly used in industry and households. Centrifugal pumps have a simple and versatile structure. With the development of high-speed engine technology, centrifugal pumps are more efficient than the era before the invention of the high-speed motor.

The use of pumps is very broad in supporting production processes ranging from large industries to the home level. The types of pumps on the market have various variants, the use of the pump is tailored to the needs. It can even be said that the presence of a pump cannot be separated in industrial life. With the wide use of pumps, a test is needed to determine the performance of the pump. Centrifugal pumps are one of the most widely used types of pumps in households. The centrifugal pump is a type of fluid transfer pump, with the working principle of converting kinetic energy (speed) into potential energy (dynamic) through an impeller that rotates in the casing. In general, a pump is considered to have good quality if it has a strong thrust and deep suction power. In more general terms the pump thrust is referred to as the pump head. The higher the head indicates the high power generated by the pump.

Judging from the assessment generally understood by the public, engineering is needed to increase the pump lift and suction strength. The reduction in lift at the pump is often called head looss. The physical meaning of the head loss is the loss of mechanical 
energy in the mass unity of the fluid. The unit head loss is a unit of length which is equivalent to one unit of energy required to move one unit of mass of fluid as high as one unit of length accordingly. The head loss calculation is based on experimental results and dimensional analysis. Head loss occurs with a bend in the pipe. With more turns, the head loss that occurs is getting bigger. (Edi Widodo, ST., MT, 2010).

\section{Research Question}

The problems in this final project are:

"How to plan a centrifugal pump using software CF Turbo 10.2".

\section{Research Limitation}

So that the problem can be fixed on its target, the problem will be focused on:

1. Does not discuss thermodynamic processes in pumps.

2. Does not address pump balancing issues.

3. Does not manufacture pumps.

4. Does not take power measurements, required torque versus rpm.

5. Do not perform finite element method analysis for water flow in the pump (Computational Fluid Dynamics).

\section{Research Objection}

The goal to be achieved in this planning is to plan a centrifugal pump with a capacity of $\mathrm{Q}=30 \mathrm{~m} 3$ / hour, pump head $\mathrm{H}=10$ meters, and shaft rotation $\mathrm{n}=1500 \mathrm{rpm}$.

\section{METHODOLOGY OF RESEARCH}

\section{Research Flowchart}

The several stages of the process that will be carried out in this centrifugal pump planning are shown in the following planning flow diagram:

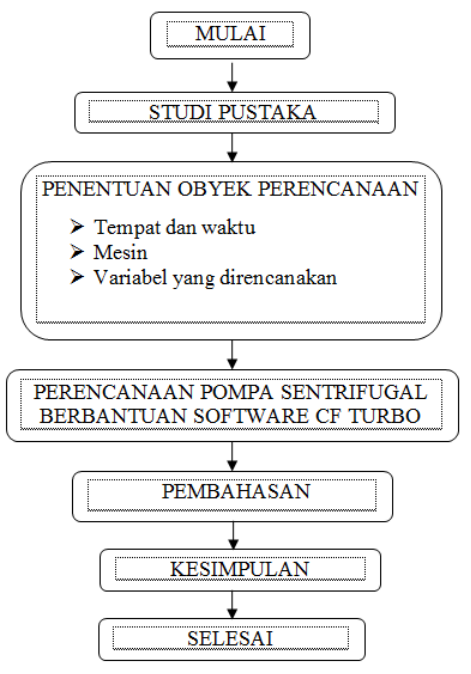

Figure 1. Research Flowchart

\section{DISCUSSION}

\section{Start and Planning}

In this plan, primary data is used, namely by measuring the centrifugal pump with the following specifications:

Table 1. Centrifugal Pump Specifications

\begin{tabular}{|l|l|}
\hline \multicolumn{1}{|c|}{ Parameter terukur } & \multicolumn{1}{c|}{ Dimensi } \\
\hline Hub diameter $(d H)$ & $25 \mathrm{~mm}$ \\
\hline Suction diameter $(d S)$ & $88 \mathrm{~mm}$ \\
\hline Impeller diameter $(d 2)$ & $178 \mathrm{~mm}$ \\
\hline Outlet width $(b 2)$ & $14 \mathrm{~mm}$ \\
\hline Pump capacity $(Q)$ & $30 \mathrm{~m}^{3} /$ hour \\
\hline Head Pompa $(H)$ & $10 \mathrm{~meter}$ \\
\hline Rotation $(n)$ & $1500 \mathrm{rpm}$ \\
\hline
\end{tabular}

In the design process of the centrifugal pump impeller section based on $\mathrm{CF}$ Turbo software, there are several steps that must be carried out sequentially. The process steps are as follows:

\section{a. Project}

This process is a process of entering the dimensions of the centrifugal pump and 
determining the impeller model according to the type of pump being analyzed. Another parameter is a design variable that can be modified to improve the performance and efficiency of the impeller while operating.

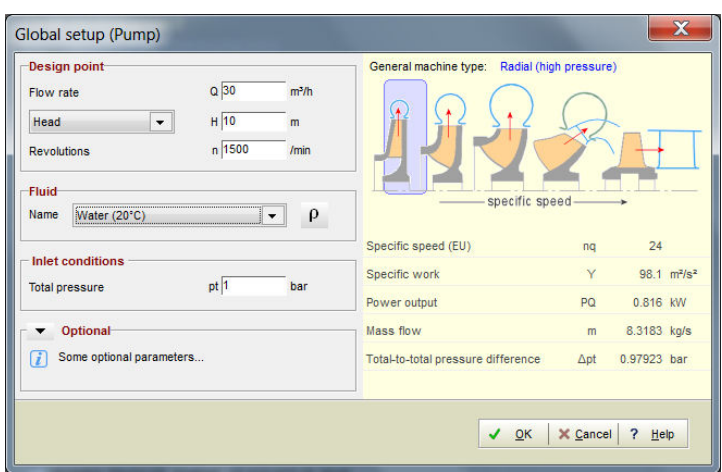

Figure 2. Global Setup

\section{b. Impeller}

This menu is used to carry out the calculation process to obtain three display variables, namely:

\section{$>$ Value}

This menu is a menu that displays the calculation results based on the centrifugal pump parameters that have been entered.

\begin{tabular}{|c|c|c|}
\hline \multicolumn{3}{|l|}{ Characteristics } \\
\hline Meridional flow coefficient & 甲m & 0.083 \\
\hline Flow coefficient & $\varphi t$ & 0.024 \\
\hline Work coefficient & $\psi$ & 1.003 \\
\hline Diameter coefficient & $\delta$ & 6.472 \\
\hline \multicolumn{3}{|l|}{ Inlet } \\
\hline Averaqe inlet velocity & $\mathrm{cms}$ & $1.6 \mathrm{~m} / \mathrm{s}$ \\
\hline Averaqe inlet velocity (net) & $\mathrm{cms}^{*}$ & $1.5 \mathrm{~m} / \mathrm{s}$ \\
\hline Inlet circ. velocity & cus & $0 \mathrm{~m} / \mathrm{s}$ \\
\hline Inlet rel. velocity & ws & $4.2 \mathrm{~m} / \mathrm{s}$ \\
\hline Inlet pressure & $\mathrm{pS}$ & 49.99 bar \\
\hline Inlet total pressure & ptS & 50 bar \\
\hline \multicolumn{3}{|l|}{ Outlet } \\
\hline Outlet mer. velocity & $\mathrm{cm} 2$ & $1.2 \mathrm{~m} / \mathrm{s}$ \\
\hline Outlet mer. velocity (net) & $\mathrm{cm}^{2}$ & $1.1 \mathrm{~m} / \mathrm{s}$ \\
\hline Outlet circ. velocity & cu2 & $8.2 \mathrm{~m} / \mathrm{s}$ \\
\hline Outlet rel. velocity & w2 & $5.9 \mathrm{~m} / \mathrm{s}$ \\
\hline Outlet peripheral speed & u2 & $14 \mathrm{~m} / \mathrm{s}$ \\
\hline Outlet pressure & p2 & 50.6 bar \\
\hline Outlet total pressure & pt2 & 51 bar \\
\hline \multicolumn{3}{|l|}{ Global values } \\
\hline Meridional deceleration & $\mathrm{cm} 2 / \mathrm{cmS}$ & 0.73 \\
\hline Relative velocity ratio S->2 & w2/wS & 1.40 \\
\hline Outlet width ratio & $\mathrm{b} 2 / \mathrm{d} 2$ & 0.08 \\
\hline Axial force & $\mathrm{Fax}$ & $489 \mathrm{~N}$ \\
\hline
\end{tabular}

Figure 3. Impeller Calculation Results

\section{Meridian}

This menu describes a sketch of the impeller section of the centrifugal pump resulting from the input dimension parameters.

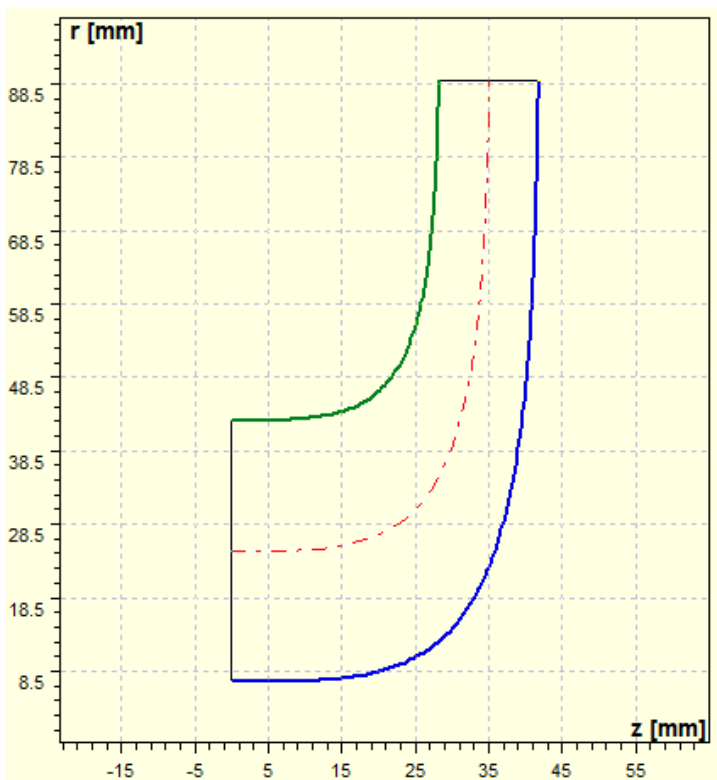

Figure 4. Impeller Cross-sectional Sketch

\section{Diagram Cordier}

The dimension parameters of the centrifugal pump entered will be able to display the appropriate impeller type. In this case the pump dimension parameters entered to produce the appropriate impeller type are radial based on specific diameter, specific speed and work coefficient. 


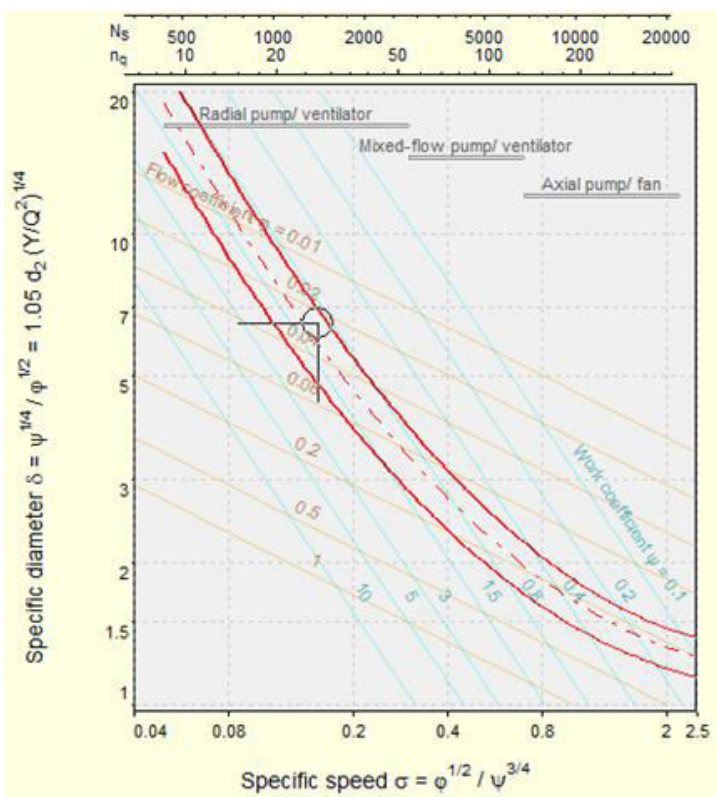

Figure 5. Impeller Cross-sectional Sketch

\section{c. Meridional Contour}

Meridional contours are second most important after impeller planning. In this menu we can change the line position according to the need to get the most pump performance. Through this menu we can see the meridional contours in 2 and 3 dimensions as well as the detailed dimensions of the impeller geometry.

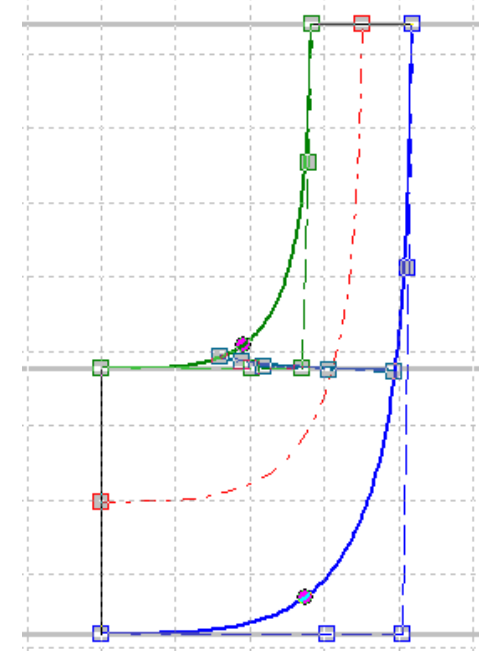

Figure 6. Meredional Contour 2D

\section{d. Blade Properties}

In the blade design process, there are two steps, namely: Blade Setup The blade setup is used to determine the number, thickness and shape of the blades in the pump.

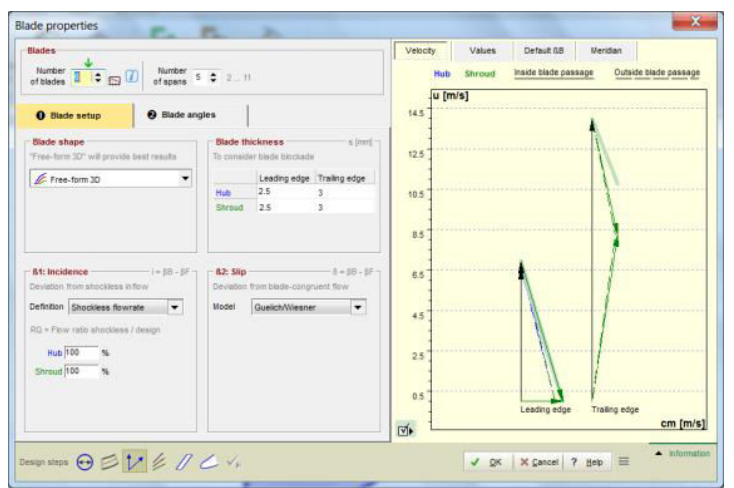

Figure 7. Blade Setup

\section{e. Blade Angles}

Average line planning depends on the number and position of meridional blade profiles called blade angles. Blade angles $\beta$ B1 and $\beta$ B2 are calculated based on the velocity triangle.

The degrees of freedom of blade planning depend on the blade shape. It should refer to the blade angles that are the result of the average line calculation.

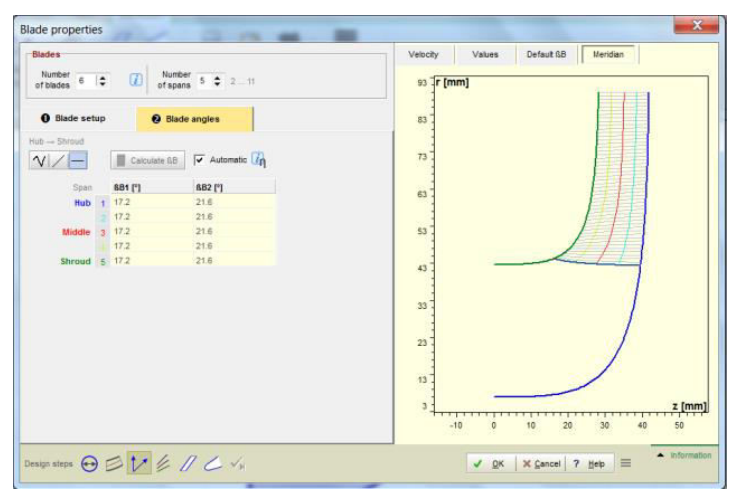

Figure 8. Blade Angels

\section{f. Blade mean line}

It is a depiction that combines blade angle, blade passage area and lean angle. Based on the combination of the three, it can be seen that not all blade profile lines have the same length. That is because the blade profile lines have a different maximum m-value. 


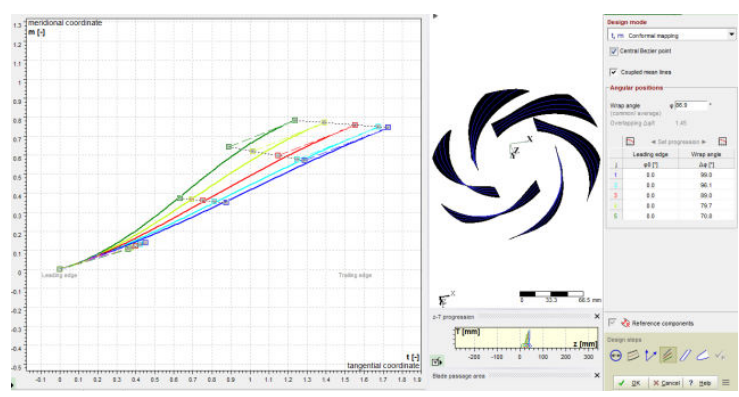

Figure 9. Blade Mean Line

\section{g. Blade profile}

To make blade profiles orthogonal blade thickness distribution for hubs and shroud profiles are used. Orthogonal blade thickness values are added to both sides of the blade to create side pressure and suction.

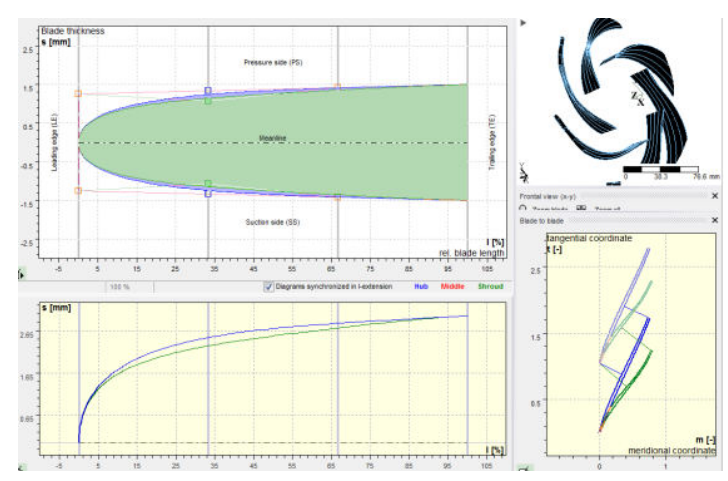

Figure 10. Blade Profile

\section{h. Blade Edges (Leading Edge)}

The blade is designed to have a blunt end and a trailing edge (the line between the endpoint of the suction and the pressure side). The blade edge is designed by determining the thickness distribution.

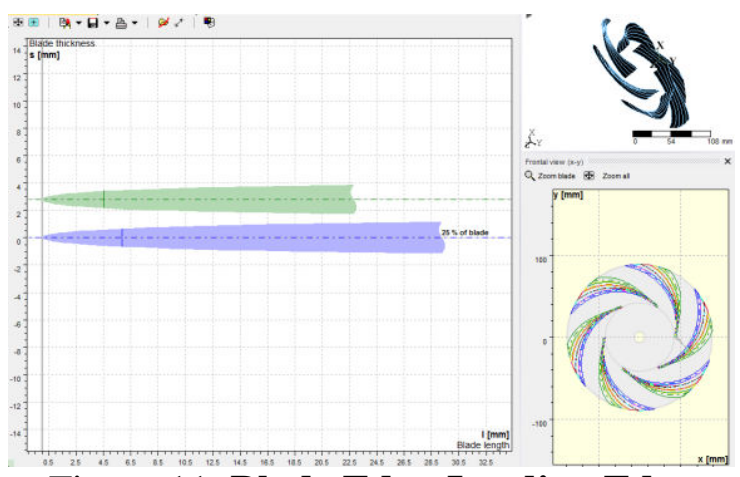

Figure 11. Blade Edge-Leading Edge

\section{i. Blade Edge (Trailing Edge)}

The trailing edge is the planned portion of the back edge of the impeller.

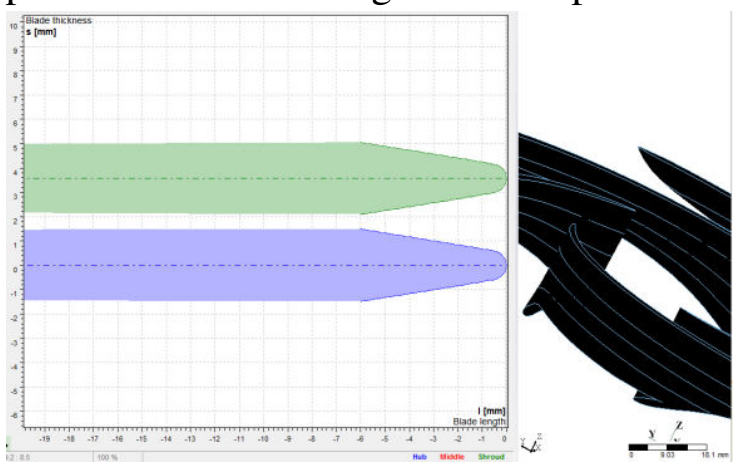

Figure 12. Blade Edge-Trailing Edge

\section{j. Volute}

The spiral area development can be calculated manually or automatically. The results of these calculations can be displayed in the form of cross-sectional images, spiral areas, diffusers and cut-water.

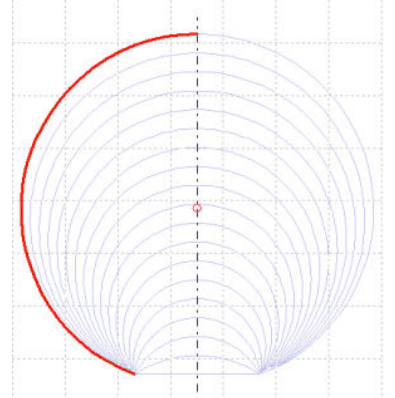

Figure 13. Volute Profile

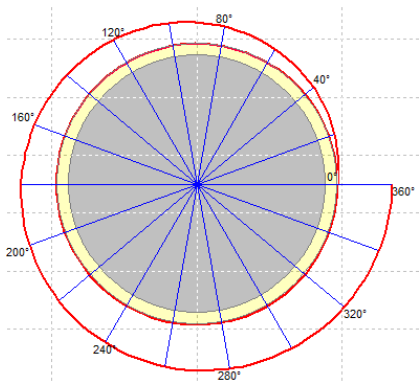

Figure 14. Spiral Area 


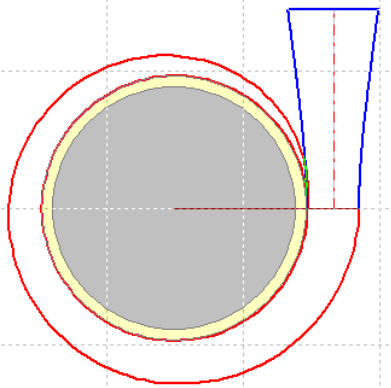

Figure 15. Diffuser

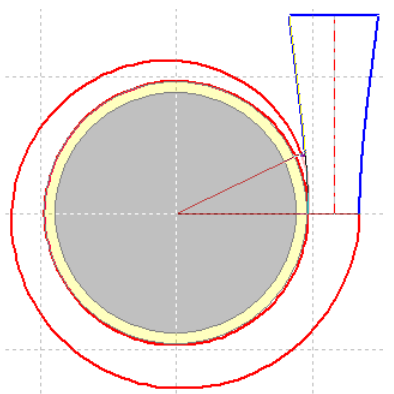

Figure 16. Cut Wear

Based on the simulation that has been carried out like the steps above, the final results of the impeller design are as follows:

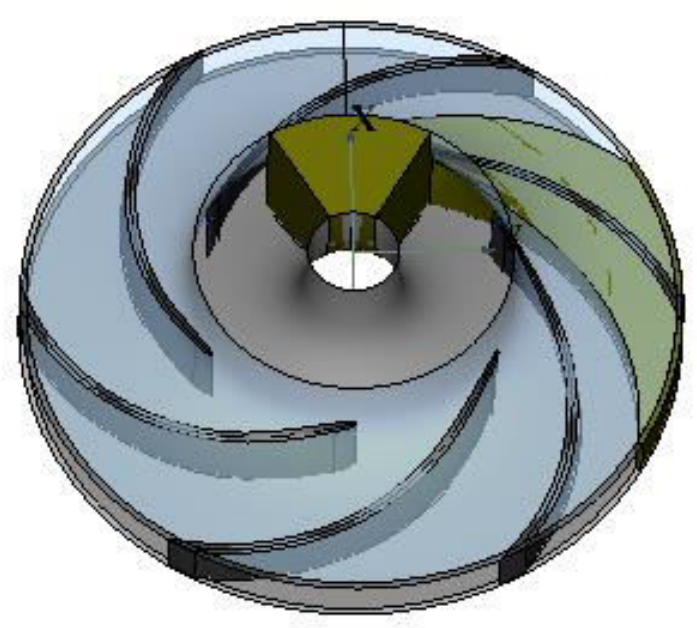

Figure 17. 3D Design of Impeller

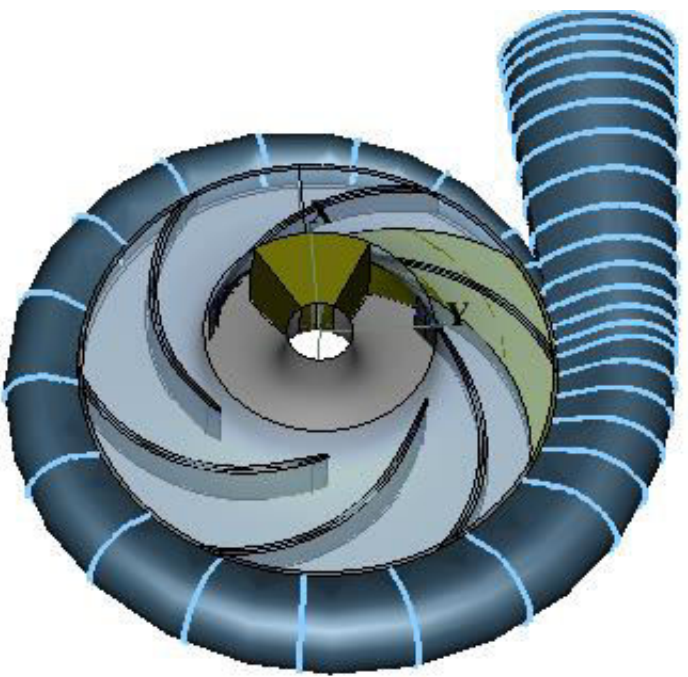

Figure 18. Impeller \& Casing

Based on the simulation that has been carried out like the steps mentioned above, the optimal parameter results are as follows:

Table 2. Software Input and Output Parameters

\begin{tabular}{|c|c|c|c|}
\hline \multicolumn{4}{|l|}{ Design Point } \\
\hline Massa flow & $\mathrm{M}$ & {$[\mathrm{kg} / \mathrm{s}]$} & 8.3183 \\
\hline Revolutions & $\mathrm{N}$ & {$[/ \mathrm{min}]$} & 1500 \\
\hline Additional hydroulic & $\eta \mathrm{h}+$ & {$[\%]$} & 100 \\
\hline Specific work & $\mathrm{Y}$ & {$\left[\mathrm{m}^{2} / \mathrm{s}^{2}\right]$} & 98,1 \\
\hline Specific speed (EU) & $\mathrm{nq}$ & & 24 \\
\hline Power output & $\mathrm{PQ}$ & {$[\mathrm{W}]$} & 816,0 \\
\hline Rotation direction & & & Right \\
\hline Swisrl number & $\mathrm{A}$ & 0 & 90 \\
\hline Flow rate & $\mathrm{Q}$ & {$\left[\mathrm{m}^{3} / \mathrm{h}\right]$} & 30 \\
\hline $\begin{array}{l}\text { Total pressure } \\
\text { difference }\end{array}$ & $\Delta \mathrm{pt}$ & [bar] & 0,97923 \\
\hline Head & $\mathrm{H}$ & {$[\mathrm{m}]$} & 10 \\
\hline \multicolumn{4}{|l|}{ Fluid Properties } \\
\hline Fluid name & & & $\begin{array}{l}\text { Water } \\
20^{\circ} \mathrm{C}\end{array}$ \\
\hline Density & $\mathrm{P}$ & {$\left[\mathrm{kg} / \mathrm{m}^{3}\right]$} & 998,2 \\
\hline \multicolumn{4}{|l|}{ Parameters } \\
\hline Hydroulic eficiency & $\eta \mathrm{h}$ & & 85,2 \\
\hline Volumetric eficiency & $\eta_{\mathrm{v}}$ & [\%] & 94,8 \\
\hline Internal eficiency & $\eta 1$ & [\%] & 77,1 \\
\hline $\begin{array}{l}\text { Mechanical } \\
\text { Eficiency }\end{array}$ & $\eta \mathrm{m}$ & [\%] & 96,9 \\
\hline Motor eficiency & Пmot & [\%] & 80 \\
\hline Stage eficiency & nst & [\%] & 74,7 \\
\hline Stage eficiency incl & $\eta \mathrm{st} *$ & [\%] & 59,8 \\
\hline $\begin{array}{l}\text { Empirical funtion for } \\
\text { dS parameter }\end{array}$ & & & $\begin{array}{l}\text { Cf Turbo } \\
\text { default }\end{array}$ \\
\hline $\begin{array}{l}\text { Empirical funtion for } \\
\text { dl parameter }\end{array}$ & & & $\begin{array}{l}\text { Cf Turbo } \\
\text { default }\end{array}$ \\
\hline
\end{tabular}


Siprianus Ndaha Ate (2019), TRANSMISI, Vol-15 No.1/ p. 369-376

\begin{tabular}{|c|c|c|c|}
\hline $\begin{array}{l}\text { Empirical funtion for } \\
\text { bl parameter }\end{array}$ & & & $\begin{array}{l}\text { Cf Turbo } \\
\text { default }\end{array}$ \\
\hline Work coeffisient & $\psi$ & {$[-]$} & 1,003 \\
\hline Outled width ratio & $\mathrm{b} 2 / \mathrm{d} 2$ & {$[-]$} & 0,076 \\
\hline $\begin{array}{l}\text { Required driving } \\
\text { power }\end{array}$ & PD & {$[\mathrm{kW]}$} & 1.1 \\
\hline Required power inch & PR & {$[k W]$} & 1,4 \\
\hline Intake coofisient & $\tau$ & {$[-]$} & 0,113 \\
\hline $\begin{array}{l}\text { Side friction } \\
\text { eficiency }\end{array}$ & $\eta_{\mathrm{s}}$ & [\%] & 95,5 \\
\hline \multicolumn{4}{|l|}{ Main dimension } \\
\hline Automatic calculator & & & Yes \\
\hline Hub diameter & $\mathrm{dH}$ & {$[\mathrm{mm}]$} & 25 \\
\hline Suction diameter & $\mathrm{dS}$ & {$[\mathrm{mm}]$} & 87,7 \\
\hline Work coefficient & $\psi$ & {$[-]$} & 1,00 \\
\hline $\begin{array}{l}\text { Average inlet } \\
\text { velocity }\end{array}$ & $\mathrm{cmS}$ & {$[\mathrm{m} / \mathrm{s}]$} & 1,58 \\
\hline $\begin{array}{l}\text { Average inlet } \\
\text { velocity }\end{array}$ & $\mathrm{cmS}^{*}$ & {$[\mathrm{~m} / \mathrm{s}]$} & 1,50 \\
\hline $\begin{array}{l}\text { Average outlet } \\
\text { velocity }\end{array}$ & $\mathrm{Cm} 2$ & {$[\mathrm{~m} / \mathrm{s}]$} & 1,16 \\
\hline $\begin{array}{l}\text { Average outlet } \\
\text { velocity inch }\end{array}$ & $\mathrm{Cm} 2^{*}$ & {$[\mathrm{~m} / \mathrm{s}]$} & 1,10 \\
\hline Impeller diameter & $\mathrm{d} 2$ & {$[\mathrm{~mm}]$} & 178,1 \\
\hline Outlet width & b2 & {$[\mathrm{m} / \mathrm{s}]$} & 13,54 \\
\hline \multicolumn{4}{|l|}{ Meridional contour } \\
\hline Desai mode & & & $\begin{array}{l}\text { Hub } \\
\text { shroud }\end{array}$ \\
\hline Axial extension max. & $\begin{array}{l}\Delta \mathrm{zM} \\
\mathrm{ax}\end{array}$ & {$[\mathrm{mm}]$} & 41,821 \\
\hline Radial extension & $\begin{array}{l}\Delta \mathrm{rM} \\
\mathrm{ax}\end{array}$ & {$[\mathrm{mm}]$} & 76,55 \\
\hline Axial extension & $\Delta \mathrm{z}$ & {$[\mathrm{mm}]$} & 35,051 \\
\hline \multicolumn{4}{|l|}{ Setup } \\
\hline Type & & & Single \\
\hline Volumetric eficiency & $\eta_{\mathrm{v}}$ & {$[-]$} & 1 \\
\hline Flow faktor & $\mathrm{FQ}$ & {$[-]$} & 1 \\
\hline \multicolumn{4}{|l|}{ Spiral Inlet } \\
\hline Diameter & $\mathrm{d}$ & {$[\mathrm{mm}]$} & 181.7 \\
\hline width & $\mathrm{b}$ & {$[\mathrm{mm}]$} & 13,54 \\
\hline \multicolumn{4}{|l|}{ Inlet } \\
\hline Hub point & & {$[\mathrm{mm}]$} & {$[41.853 ; 90.784]$} \\
\hline Shroud point & & {$[\mathrm{mm}]$} & {$[28.328 ; 90.784]$} \\
\hline Center point & & {$[\mathrm{mm}]$} & [35.091;90.784] \\
\hline Offset hub & & {$[\mathrm{mm}]$} & {$[0.0071089 ; 0]$} \\
\hline Offset Shroud & & {$[\mathrm{mm}]$} & {$[-0.0071089 ; 0]$} \\
\hline Offset center & & {$[\mathrm{mm}]$} & {$[0 ; 0]$} \\
\hline Width & $\mathrm{b}$ & {$[\mathrm{mm}]$} & $13 ; 54$ \\
\hline Angel mode & & & Axial \\
\hline \multicolumn{4}{|l|}{ Cross section } \\
\hline Cross section & & & RadiusBased \\
\hline Angel & $\varphi$ & {$\left[{ }^{0}\right]$} & 360 \\
\hline Main radius & $\mathrm{R}$ & {$[\mathrm{mm}]$} & 0 \\
\hline Cone angle & $\sigma$ & {$\left[{ }^{0}\right]$} & 30 \\
\hline Base height & $\mathrm{h}$ & {$[\mathrm{mm}]$} & 0 \\
\hline Base radius & $\mathrm{BR}$ & {$[\mathrm{mm}]$} & 0 \\
\hline Corner radius & $\mathrm{RC}$ & {$[\mathrm{mm}]$} & 0 \\
\hline
\end{tabular}

\begin{tabular}{|c|c|c|c|}
\hline \multicolumn{4}{|c|}{ Spiral areas geometry } \\
\hline Design rule & & & fleiderer \\
\hline Swirl exponent & & {$[-]$} & 1 \\
\hline $\begin{array}{l}\text { Automatic flow } \\
\text { properties }\end{array}$ & & & No \\
\hline Ref. velocity & & {$[\mathrm{m} / \mathrm{s}]$} & 8.0742 \\
\hline Volume flow & & {$\left[\mathrm{m}^{3} / \mathrm{h}\right]$} & 0.0083333 \\
\hline Wrap angel & $\Phi$ & {$\left[{ }^{0}\right]$} & 360 \\
\hline Dicharge area & $\mathrm{A}$ & {$\left[\mathrm{mm}^{2}\right]$} & 1284.5 \\
\hline \multicolumn{4}{|l|}{ Diffuser } \\
\hline Direction & & & Tangensial \\
\hline Eccentricty & $\Delta \mathrm{x}$ & {$[\mathrm{mm}]$} & 0 \\
\hline Eccentricty mode & & & Centric \\
\hline Heigt & $\mathrm{H}$ & {$[\mathrm{mm}]$} & 136.18 \\
\hline Center distance & $\mathrm{C}$ & {$[\mathrm{mm}]$} & 113.25 \\
\hline Start angel & $\Phi^{0}$ & {$\left[{ }^{0}\right]$} & 0 \\
\hline \multicolumn{4}{|l|}{ Endcross section } \\
\hline Type & & & Circle \\
\hline Exit diameter & $\mathrm{D}$ & {$[\mathrm{mm}]$} & 66.2 \\
\hline $\begin{array}{l}\text { End cross section } \\
\text { position }\end{array}$ & & [\%] & 100 \\
\hline Lenght & $\mathrm{L}$ & {$[\mathrm{mm}]$} & 136.18 \\
\hline Cone angel & $\Theta$ & {$\left[{ }^{0}\right]$} & 5.4 \\
\hline \multicolumn{4}{|l|}{ Cut water } \\
\hline Mode & & & Simple \\
\hline Position & $\Phi \mathrm{c}, 0$ & {$\left[{ }^{0}\right]$} & 37.3 \\
\hline Equiv. Diff. diam. & $\mathrm{D}$ & {$[\mathrm{mm}]$} & 53.768 \\
\hline Cross section area & A & {$\left[\mathrm{mm}^{2}\right]$} & 2270.6 \\
\hline Radial offset & & {$[\mathrm{mm}]$} & 0 \\
\hline Side position left & & & 0.288 \\
\hline Side posetion right & & & 0.288 \\
\hline Rounded edges & & & No \\
\hline \multicolumn{4}{|l|}{ Cut water angel } \\
\hline Inner & $\alpha \operatorname{lnn}$ & {$\left[{ }^{0}\right]$} & 5.4708 \\
\hline Outer & $\alpha$ Out & {$\left[{ }^{0}\right]$} & 32.328 \\
\hline Average & $\alpha \mathrm{Avg}$ & {$\left[{ }^{0}\right]$} & 16.622 \\
\hline \multicolumn{4}{|c|}{ Cut water diameter } \\
\hline Inner & dlnn & {$[\mathrm{mm}]$} & 200.31 \\
\hline Outer & dOut & {$[\mathrm{mm}]$} & 210.9 \\
\hline Average & dAvg & {$[\mathrm{mm}]$} & 202.42 \\
\hline Minimal & dMin & {$[\mathrm{mm}]$} & 200.02 \\
\hline
\end{tabular}

\section{CONCLUSION}

In planning a centrifugal pump with the aid of CF Turbo 10.2 software, a fivestage water pump can be planned.

The pump that is planned is as shown in the image below with the following specifications: 

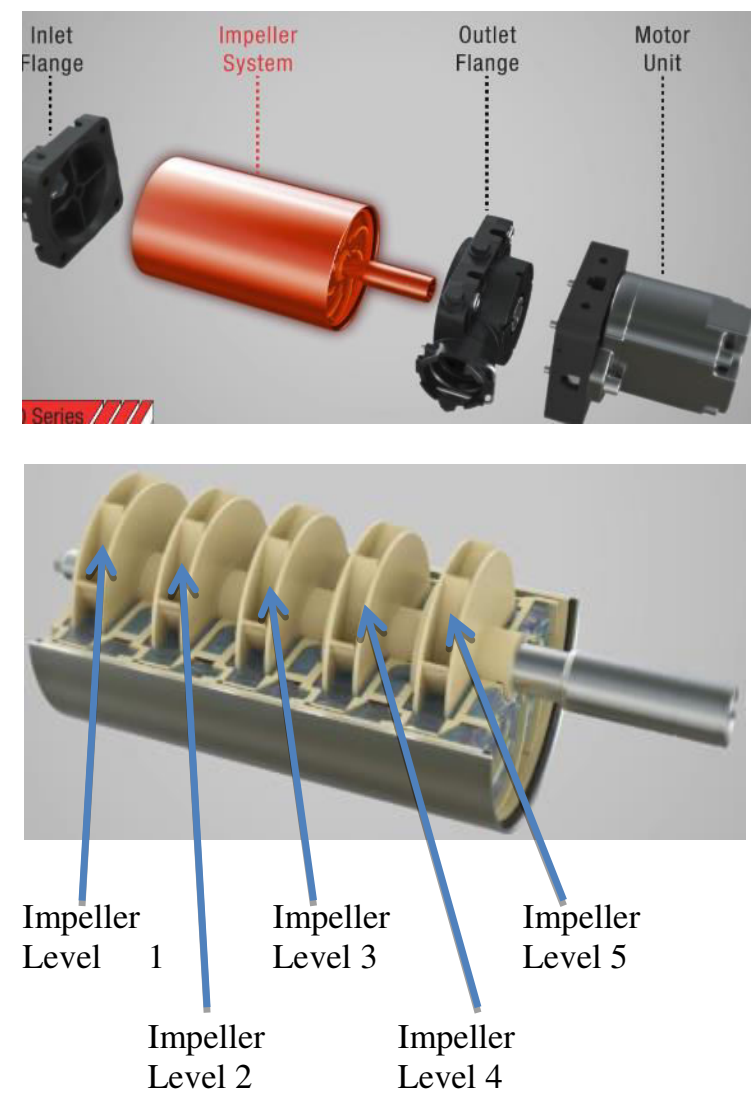

Table 3. Pump Specification

\begin{tabular}{|c|c|c|c|c|c|}
\hline & Level 1 & Level 2 & Level 3 & Level 4 & Level 5 \\
\hline $\begin{array}{c}\text { Debit } \\
\left(\mathrm{m}^{3} / \mathrm{jam}\right)\end{array}$ & 30 & 30 & 30 & 30 & 30 \\
\hline $\begin{array}{c}\text { Input } \\
\text { pressure } \\
\text { (bar) }\end{array}$ & 1 & 10 & 20 & 30 & 40 \\
\hline $\begin{array}{c}\text { Output } \\
\text { pressure } \\
\text { (bar) }\end{array}$ & 10 & 20 & 30 & 40 & 50 \\
\hline $\begin{array}{c}\text { Spindle } \\
\text { rotation } \\
\text { (rpm) }\end{array}$ & 1500 & 1500 & 1500 & 1500 & 1500 \\
\hline $\begin{array}{c}\text { Motor } \\
\text { power } \\
(\mathrm{kW})\end{array}$ & 1,4 & 1,4 & 1,4 & 1,4 & 1,4 \\
\hline
\end{tabular}

Based on the simulations that have been carried out based on the dimensional parameters of the centrifugal pump with the technical specifications mentioned above, it can be concluded that:

1. This type of centrifugal pump impeller is a radial impeller.

2. Simulations that have been carried out on a centrifugal pump impeller have produced the most optimal parameters.

\section{REFERENCES}

Darto, I Made Sunada (2017), Analisis kinerja geometrik impeller pompa sentrifugal berbasis perangkat lunak, Transmisi, volxii edisi-2/ hal. 87-94.

Edi Widodo, ST, MT, 2016, Rekayasa Performansi Pompa Sentrifugal Untuk Menurunkan Head Loss, Laporan Akhir Penelitian Dosen Pemula, Universitas Muhammadiyah Sidoarjo.

Erwin Martianis ${ }^{1}$ Ikhwansyah Isranuri ${ }^{2}$ Indra $^{3}$, 2012, Analisa Getaran Pada Pompa Sentrifugal Sistem Penyambungan Kopling Sabuk Untuk Monitoring Kondisi, Jurnal Dinamis, Volume II, No.10, Januari 2012, Teknik Mesin USU

Prihadi Nikosai TBS dan Irfan Syarief Arief, 2015, Optimasi Desain Impeller Pompa Sentrifugal Menggunakan Pendekatan CFD, Jurnal Sains Dan Seni ITS Vol. 4, No.2, (2015), Fakultas Teknologi Kelautan, Jurusan Teknik Sistem Perkapalan, Institut Teknologi Sepuluh Nopember (ITS).

Series Information SKP, Strobl Pumpen, Multi Stage Pumps, Series SKP.

Tahara Haruo $^{1}$, dan Sularso ${ }^{2}, 1991$, Pompa dan kompresor: Pemilihan,Pemakaian dan Pemeliharaan. Penerbit: Pradnya Paramita, Jakarta.

Thoharudin $^{1}$, Arif Setyo Nugroho ${ }^{2}$, Stefanus Unjanto $^{3}$, 2014, Optimasi Tinggi Tekan Dan Efisiensi Pompa Sentrifugal Dengan Perubahan Jumlah Sudu Impeler Dan Sudut-Sudu Keluar Impeler (B2) Menggunakan Simulasi Computational Fluid Dynamics, Teknologi (SNAST) 2014 Yogyakarta ${ }^{1,2,3}$ Jurusan Teknik Mesin.

Tri Yanto, 2016, Perencanaan Impeller Pompa Sentrifugal dengan Kapasitas 58 liter/detik Head 70 m dengan Putaran 2950 rpm Penggerak Motor Listrik.

Yogi Candra, 2016, Perawatan \& Perbaikan Pompa Sentrifugal, Untuk Pemasok Chlorine Pada Sistem Chlorination Plant Pltgu Up Muara Tawar Kabupaten Bekasi, Tugas Akhir, Jurusan Teknik Mesin Politeknik Negeri Padang. 